\title{
Theoretical and experimental research on the friction coefficient of water lubricated bearing with consideration of wall slip effects
}

\author{
Zhongliang Xie ${ }^{1}$, Zhu-shi RaO ${ }^{1, a}$, TA-NA ${ }^{1}$, Ling LiU ${ }^{1}$ And Rugang Chen ${ }^{2}$ \\ 1 State Key Laboratory of Vibration, Shock and Noise, Shanghai JiaoTong University, Shanghai 200240, P.R. China \\ 2 China Ship Development and Design Center, Wuhan 430064, P.R. China
}

Received 19 January 2015, Accepted 27 May 2015

\begin{abstract}
Researches of the nature of friction coefficient of water lubricated bearing are carried out in this paper. Based on the experimental results of composite material bearing under hydrodynamic lubrication by water, we calculate the Stribeck curves as the function of load and speed, deviate the modified Reynolds equation considering wall slip effects in Cartesian coordinate system, put forward the essential model of friction coefficient, the composition of friction coefficient and essential changing correlation between them. Numerical results are in good agreement with experimental results, which verifies existence of wall slip. Researches indicate that there is always an appropriate working condition which friction coefficient is minimum. Comparison of the theoretical and experimental results shows that they are consistent on the overall trend but still exist some deviations under certain operating conditions. Reasonable explanations are given to illustrate the correctness of theoretical model.
\end{abstract}

Key words: Water lubricated bearing / nature of friction coefficient / wall slip effect / solid contact effect and fluid viscous effect / experimental research

\section{Introduction}

Water lubricated bearing has great potential in environmental protection, energy conservation as well as sustainable development and thus has been widely used in ships, water pumps and other mechanical systems for its advantages of no pollution, wide source, safety and fire resistance, etc. It can effectively reduce the wear, noise and power consumption due to the relative movements of friction pair material $[1,2]$. Its lubrication performance, reliability and safety have important effects on the safe and stable operation of mechanical system, therefore, the research and improvement of lubrication performance are of vital directive significance for the promotion and application of water lubricated bearing.

The Stribeck curve plays an important role in identifying boundary, mixed, elasto-hydrodynamic and hydrodynamic lubrication regime as well as tribological properties in water lubricated bearing [3-7]. Presently, most of the researchers are interested in investigating the theories and experiments of hydrodynamic lubrication, many scholars have done much in this field [8-13]. For example, Alex de Kraker [14] adopts an ideal asperity contact model together with an effective film thickness formulation to compute Stribeck curve at constant load for water

${ }^{a}$ Corresponding author: zsrao@sjtu.edu.cn lubricated journal bearing. Computed Stribeck curves are presented and sensitivity of the computed Stribeck curve and minimum film thickness with respect to the design parameters (such as: clearance, surface roughness, load) and the material parameters (such as: modulus of elasticity, surface hardness) are also systematically researched in this paper. $\mathrm{Lu}$ [5] reports the Stribeck-type behavior results of a series of experiments under various oil inlet temperatures and loads, the results are also compared with simulations of the Stribeck curves using the approaches presented in references $[4,6]$. The theoretical verifications presented in this paper are related to mixed lubrication regime and elasto-hydrodynamic lubrication regime (EHL), where the Bair-Winer model is adopted to describe the shear stress of the lubricant. Kalin [15] investigates the Stribeck curve and the bearing lubrication design for non-fully wetted surfaces, and has experimentally verified the friction behavior by using fully wetted and non-fully wetted model surfaces in different contact configurations. So far, researches of water lubricated bearing are mainly about the tribology behaviors of sliding surfaces of silicon nitride, polymer or rubber in water or sea water, but very few of them penetrate into the nature of bearing characteristics including the friction force and the correlation between the friction coefficient and the lubrication state as well as the lubrication mechanism. 


\section{Nomenclature}

\begin{tabular}{|c|c|c|c|}
\hline$\tau$ & Shear stress & & \\
\hline$\mu$ & Viscosity & $l_{x}, l_{y}$ & Length of surface 1 , surface 2 \\
\hline$u_{c}$ & Critical slip velocity & $u_{x}, u_{y}$ & Velocity component in $x, y$-direction \\
\hline$\tau_{c}$ & Critical shear stress & $q_{x}, q_{y}$ & Volumetric flow rate in $x, y$-direction \\
\hline$u_{s}$ & Wall slip velocity & $\sigma$ & Aspect ratio, $l_{x} / l_{y}$ \\
\hline$p$ & Film pressure & $H, F, P, U, W$ & Dimensionless variables \\
\hline$p_{a}$ & Ambient pressure & $L, D$ & Width and diameter of the test bearing \\
\hline$h$ & Film thickness & $z$ & Cross-film or axial coordinate \\
\hline$h_{\min }$ & Minimum film thickness & $N$ & Rotating speed of journal \\
\hline$\lambda$ & Film thickness ratio & $R$ & Force arm \\
\hline$f$ & Friction coefficient & $G$ & Gravity of the bearing \\
\hline$c$ & Radical clearance & $T$ & Tangential force \\
\hline$e$ & Eccentricity & $\mathrm{Fr}$ & Radial load \\
\hline$\varepsilon$ & Eccentricity ratio, $e / c$ & $R_{q A}, R_{q B}$ & RMS surface roughness of the contacting surface $A$ and $B$ \\
\hline
\end{tabular}

Fluid flow boundary condition is one of the most important factors which determines the fluid dynamic characteristics. All along, the classical fluid mechanics, the lubrication theory and scientific researches utilize the "no slippage boundary conditions", namely: no wall slip occurs in the solid-liquid interface, and the relative speed of motion between fluid molecules on the solid surface and the solid interface is zero. This hypothesis is verified by experiments to a macro sense, and has been widely used in theoretical and experimental researches in fluid dynamic problems. However, in recent years, with the development of micro-nanometer science, technology and related fields as well as the help of some modern measurement technologies, such as the atomic force microscope (AFM), surface force apparatus (SFA), micro-particle image velocimeter $(\mu$-PIV), near field laser velocimeter (NFLV) and the molecular dynamics simulation (MDS) etc., researchers find that no slip boundary condition is no longer applicable under certain conditions, namely: boundary slip may occur in many instances [16-21]. Therefore, boundary slip phenomenon affects the fluid dynamic behaviors. So, the influence of wall slip on the lubrication performance is gaining more and more attention. For instance, Spikes et al. [22], analyze the influence of slippage on the fluid dynamic behaviors when the wall slip phenomenon occurs on the static slider surface, finding that the bearing load capacity is exactly half of the case with no slippage when the limiting shear stress equals to zero, but the corresponding friction is reduced by several orders of magnitude. Aurelian [23] investigates wall slip effects on elasto-hydrodynamic journal bearings and the study extended to the influence of wall slip in highly loaded compliant bearings for steady-state and dynamical load conditions. It also predicts that well-chosen slip/no-slip surface pattern can considerably improve the bearing behavior and largely justify future numerical and experimental works. Zhang [24] presents the development of a numerical model for high speed and water lubricated journal bearings with different boundary slip arrangements, and obtains the conclusion that a suitable combination of slip/no-slip surfaces on the sleeve of a journal bearing may enable improvement of the tribological performances through suppressing the occurrence of cavitation, enhance the load bearing capacity and reduce the interfacial friction between bearing sleeve and shaft. However, very few of the researches penetrate into the nature of bearing characteristics as well as the lubrication mechanism considering the wall slip effects. The effect of wall slip on the lubrication performance is not very clear, therefore, researches on the wall slip effects should be further studied.

In the present research, on the basement of experiment of composite material bearing under hydrodynamic lubrication by water, we calculate the Stribeck curves as the function of load and speed, deviate the modified Reynolds equation considering wall slip effects in Cartesian coordinate system, develop the essential model of friction coefficient, analyze the composition of friction coefficient and the essential changing correlation between them in water lubricated bearing. The research results are of important guiding significance for the structure design and optimization of water lubricated bearing.

\section{Theoretical considerations}

\subsection{Theoretical basis of wall slip}

The hypothesis in the classical lubrication theory, which assumes the fluid velocity on the solid interface is the same with the velocity of the solid surface, regardless of its magnitude or direction, is widely exploited in the vast majority of the theoretical researches and the experimental studies in lubrication problems. This is an important prerequisite for the establishment of the classical Reynolds equation. Modern bearing always works in extreme conditions: low speed, heavy load and very narrow clearance. Also these bearings are made of polymer materials and are lubricated by unconventional lubricants. Therefore wall slip phenomenon becomes more and more common in the lubrication process.

Modern research [25] on the lubricant rheology shows that: wall slip effect is closely related to the interface 


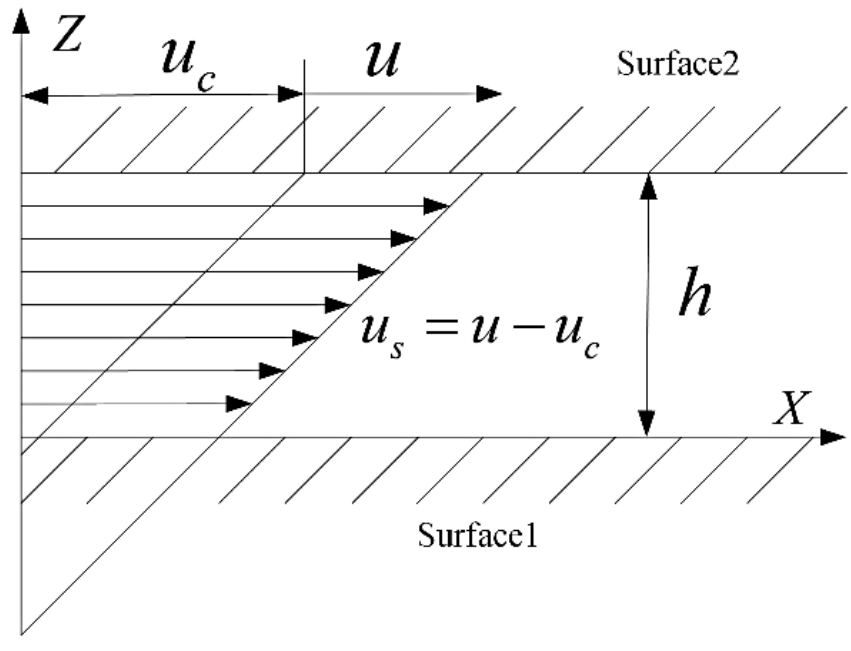

Fig. 1. Schematic of wall slip model.

shear strength. Just as the plastic flow in solid mechanics, lubricants also have a limiting shear stress. The liquid molecules will slip along the solid surface and lubricant will show the characteristics of plastic solid when the interface shear stress reaches the limiting shear strength of the friction pair material. Research [26] shows that wall slip effect only occurs in the surface with smaller limiting shear strength when there exists relative motion between two surfaces with different limiting shear strength.

Figure 1 shows the schematic of wall slip model of water lubricated bearing. Suppose that surface 1 is parallel to surface 2 , surface 2 moves with speed $u$ in the $X$-direction, while surface 1 is stationary. The limiting shear stress on surface 2 exceeds that of surface 1 , thus, wall slip phenomenon only occurs on surface 1. Assuming that when the speed meets the criteria $u=u_{c}$, surface 1 just reaches the critical state of wall slip, this corresponds to the interface limiting shear stress or limiting shear strength, correspondingly, the critical shear stress $\tau_{c}$ is:

$$
\tau_{c}=v \frac{u_{c}}{h}
$$

where $v$ is the dynamic viscosity of lubricant; $u_{c}$ the critical slip velocity; $h$ the film thickness.

With the increase of velocity on surface 2, wall slip phenomenon starts to occur in surface 1, the wall slip velocity $u_{s}$ is :

$$
u_{s}=u-u_{c}
$$

If there is no wall slip phenomenon on any of the surface, the wall slip velocity equals to zero, i.e. $u_{s}=0$. When wall slip phenomenon begins to occur, the wall slip velocity equals to the difference between the velocity of surface 2 and the critical slip velocity, i.e. $u_{s}=u-u_{c}$. One thing to note is that, the fluid velocity means the average of all the fluid molecules. When the velocity meets the requirement $u>u_{c}$, we can not say that all the lubricant molecules on the surface occur to slip.

Some of the literatures [23, 27-30] have proposed a simple criterion to distinguish whether wall slip effect has
Table 1. Interfacial tension of commonly used materials.

\begin{tabular}{cc}
\hline Material & $\begin{array}{c}\text { Interfacial tension/ } \\
\times 10^{-3} \mathrm{~N}^{-1}\end{array}$ \\
\hline Oil Based Lubricants & 30 \\
Water & 73 \\
Metal & 500 \\
Teflon & 18 \\
Nitrile Butadiene Rubber & 52.6 \\
\hline
\end{tabular}

occurred on the solid-liquid interface through theoretical and experimental researches. When the interface tension of the lubricant medium becomes bigger than that of the friction pair material, namely, adhesion fracture occurs before the cohesive fracture of molecular bonds, then the wall slip is very likely to happen.

Table 1 shows the interfacial tension of commonly used materials. It can be seen from the table that compared to oil lubricated bearing, the interfacial tension of water lubricated rubber bearing is much smaller, adhesion fracture of water molecular bonds occurs more easily, and therefore wall slip phenomenon is inclined to occur. Similarly, wall slip phenomenon also exists on the solidliquid interface of water lubricated Teflon bearing. That is to say, the influence of wall slip phenomenon should be fully considered in the numerical simulation and experimental research on water lubricated non-metallic or polymer friction pair material bearing. The modified Reynolds equation considering wall slip effects may more exactly describe the hydrodynamic characteristics of the water lubricated polymer bearings.

\subsection{Derivation of modified Reynolds equation}

The modified Reynolds equation considering wall slip is derived in Cartesian coordinate system based on the above theoretical analysis. The schematic of water lubricated bearing configuration is shown in Figures $2 \mathrm{a}$ and $2 \mathrm{~b}$, and the simplified schematic of water lubricated bearing configuration in Cartesian coordinate system is shown in Figure 2c. Surface 1 moves with speed $u$ in positive $x$ direction, while surface 2 is stationary. Wall slip phenomenon occurs on surface 1 , its critical limiting shear stress is $\tau_{c}$, and the critical slip velocity is $u_{c}$. Assuming the film thickness $h$ can vary in the $x, y$ direction, but is small enough in $z$ direction so that the lubrication approximation is valid and the transient effect in the Navier-Stokes can be ignored, i.e. the water lubricated bearing is in the state of stable operation. Thermal effects are not considered in the analysis, as the isothermal condition, the viscosity and density of water are regarded as constant, without any changes with the temperature and pressure in the film thickness direction. In addition to the isothermal condition, derivation of modified Reynolds equation also needs the following hypothesis:

(1) Ignoring the volume force, such as gravity or magnetic force.

(2) Neglecting the velocity component in the water film thickness direction as the film is very thin, i.e. $\frac{\partial h}{\partial t}=0$. 


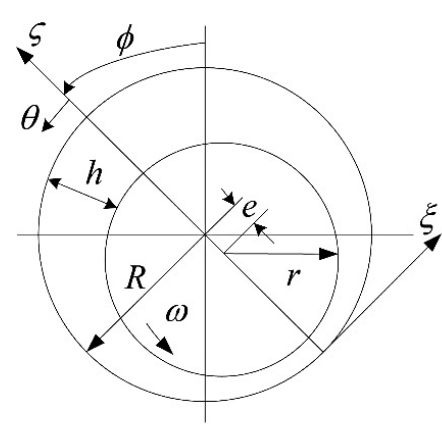

(a)

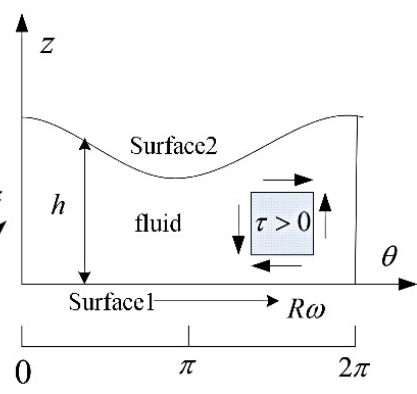

(b)

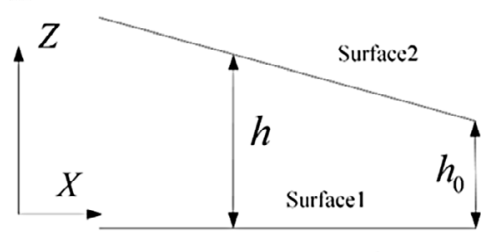

(c)

Fig. 2. Schematic of water lubricated bearing configuration.

(3) Neglecting the pressure variation in the water film thickness direction.

(4) Compared to the water film thickness, the radius of curvature of the bearing supporting surface is rather bigger. Therefore, neglecting the changes of velocity direction caused by the curvature of bearing supporting surface.

(5) Neglecting the influence of inertial force effect, the inertial force caused by the fluid acceleration as well as the centrifugal force caused by the fluid film bending.

Using the above assumption, the $x$-component of the Navier-Stokes equation becomes:

$$
-\frac{\partial p}{\partial x}+\eta \frac{\partial^{2} u_{x}}{\partial z^{2}}=0
$$

With the boundary conditions:

Surface 1:

$$
z=0, u_{x}=u
$$

Surface 2:

$$
z=h, u_{x}=u_{s}
$$

For the solution of Equation (3), subject to the boundary conditions Equations (4) and (5), we can get the velocity component in $x$ direction:

$$
u_{x}=\frac{1}{2 \mu} \frac{\partial p}{\partial x} z^{2}+\left(\frac{u-u_{s}}{h}-\frac{h}{2 \mu} \frac{\partial p}{\partial x}\right) z+u_{s}
$$

Similarly, the velocity component in $y$ direction is:

$$
u_{y}=\frac{1}{2 \mu} \frac{\partial p}{\partial x} z^{2}-\frac{h}{2 \mu} \frac{\partial p}{\partial x} z
$$

The volumetric flow rate per unit length in $x$ direction is:

$$
q_{x}=\int_{0}^{h} u_{x} \mathrm{~d} z=-\frac{h^{3}}{12 \mu} \frac{\partial p}{\partial x}+\frac{\left(u+u_{s}\right) h}{2}
$$

Similarly, the volumetric flow rate per unit length in $y$ direction is:

$$
q_{y}=\int_{0}^{h} u_{y} \mathrm{~d} z=-\frac{h^{3}}{12 \mu} \frac{\partial p}{\partial y}
$$

Conservation of mass requires:

$$
\frac{\partial q_{x}}{\partial x}+\frac{\partial q_{y}}{\partial y}=0
$$

Insert Equations (8) and (9) into Equation (10), we can get:

$$
\begin{aligned}
\frac{\partial}{\partial x}\left(h^{3} \frac{\partial p}{\partial x}\right)+\frac{\partial}{\partial y}\left(h^{3} \frac{\partial p}{\partial y}\right)= & 6 \mu\left(u+u_{s}\right) \frac{\partial h}{\partial x} \\
& +6 \mu h \frac{\partial\left(u+u_{s}\right)}{\partial x}
\end{aligned}
$$

Equation (11) is non-dimensionalized by defining the following dimensionless variables and parameters:

$$
\begin{aligned}
& X=\frac{x}{l_{x}}, Y=\frac{y}{l_{y}}, P=\frac{p}{p_{a}}, H=\frac{h}{h_{0}}, \sigma=\frac{l_{x}}{l_{y}} \\
& U=\frac{6 \mu\left(u+u_{s}\right)}{h_{0}^{2} p_{a}}, W=\frac{6 \mu H}{h_{0} p_{a}}
\end{aligned}
$$

The resulting dimensionless modified Reynolds equation becomes:

$\frac{\partial}{\partial X}\left(H^{3} \frac{\partial P}{\partial X}\right)+\sigma^{2} \frac{\partial}{\partial Y}\left(H^{3} \frac{\partial p}{\partial Y}\right)=U \frac{\partial H}{\partial X}+W \frac{\partial\left(u+u_{s}\right)}{\partial X}$

where $U \frac{\partial H}{\partial X}$ is the hydrodynamic effect term and $W \frac{\partial\left(u+u_{s}\right)}{\partial X}$ is the wall slip effects term.

\subsection{Load balance equation}

The actual water lubricated bearing is usually with longitudinal lubrication grooves. Compared to the lubrication oil, water lubricated bearing works in poor operating conditions. In the ocean or inland river environment, the lubricating water usually contains silt or other small particles, sometimes even a large quantity. Silt and other small particles will increase friction and wear, sometimes may even broke the lubricating environment. The main functions of longitudinal lubrication grooves are excluding small particles from the lubrication interface when the journal is rotating, so as not to affect the lubricating conditions of the bearing. Therefore, in the test part of the paper, we use bearing with guiding grooves to verify the theory.

Figure 3 gives the position of longitudinal lubrication grooves in relation to external load. For given external load conditions, the externally applied load is shared between fluid and solid effect at various operating conditions. When the journal is rotating at speed $N$, the static equilibrium position of the journal center $O_{j}$ can be found by equating the hydrodynamic forces and solid contact forces with the external load. The magnitude of the resultant force of $\vec{F}_{\text {fluid }}$ and $\vec{F}$ solid equals to the external vertical load $\vec{F}$ external. The direction is upward $Z$ direction. 


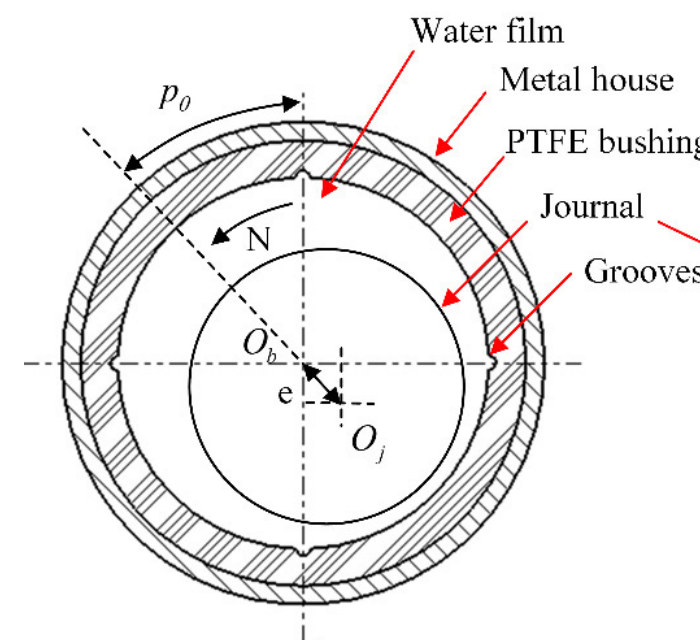

(a)

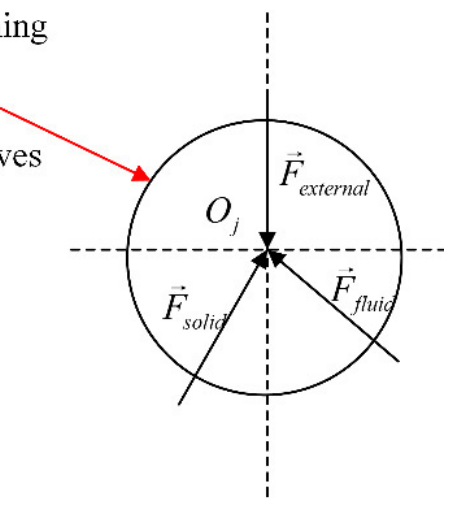

(b)

Fig. 3. Position of grooves in relation to external load.

\subsection{Boundary conditions}

The lubrication theory in the present work is general and applicable to such bearings. For water lubricated bearings with or without lubrication grooves, only the boundary condition is different. For bearing with guiding grooves, the corresponding boundary conditions are slightly more complicated than bearing without grooves, other parameters and calculating methods are almost the same. The natural rupture boundary conditions, namely the Reynolds boundary conditions, are exploited to solve the pressure distribution and film thickness distribution of water film in this paper. Reynolds boundary conditions regard water film as discontinuous, and the termination of water film pressure as some kind of natural fracture phenomenon, i.e. water film will fracture after the minimum water film thickness at an angle of $Y_{2}$, which can be written as:

$$
\begin{aligned}
& Y=0, P=P_{a} \\
& Y=Y_{2}, P=P_{a}, \frac{\mathrm{d} P}{\mathrm{~d} \theta}=0 \\
& 0<Y<Y_{2}, P=P(\theta) \\
& Y_{2}<Y<2 \pi, P=P_{a}, \frac{\mathrm{d} P}{\mathrm{~d} \theta}=0
\end{aligned}
$$

The modified Reynolds equation can be solved numerically by exploiting the finite difference method (FDM) [31], through the discretization of the partial differential coefficients using two order central difference regime. After we make iterative calculation using the Gauss-Seidel iterative method, and finally obtain the pressure distribution and the film thickness distribution. Iteration continues until the solution converges, then the lubrication parameters such as the friction coefficient can be obtained by double numerical integration over the pressure distribution area, the concrete solving process can be get in references $[32,33]$.
The dimensionless bearing friction coefficient is attained by integrating the shear stress distribution over the whole surface area. Friction force and friction coefficient can be calculated as following:

$$
\begin{aligned}
F_{f}= & F_{n}+F_{t}=\int_{0}^{2 \pi} \int_{-L / 2}^{L / 2} \frac{h}{2} \frac{\partial p}{\partial Y} \mathrm{~d} X \mathrm{~d} Y \\
& +\int_{0}^{2 \pi} \int_{-L / 2}^{L / 2} \frac{\mu r^{2} \omega}{h} \mathrm{~d} X \mathrm{~d} Y \\
f= & \frac{F_{f}}{F}
\end{aligned}
$$

where $F$ is the resultant force, $F_{f}$ is the friction force. The parameters for the test bearing are shown in Tables 2 and 3, friction coefficients calculated will be shown in Section 4.3.

In fact, $F_{f}$ is just the friction force caused by the liquid viscous shear force. In the actual bearing system, the friction coefficient is more complex. It not only includes the liquid viscous shear force, but also includes the friction due to the solid effect. Therefore, it is necessary to analyze the essence of the friction coefficient in water lubricated bearing.

\subsection{Lubrication states diagram}

Generally speaking, friction coefficient has a direct relationship with the bearing lubrication regime or the lubrication state, and the lubrication regime has a close relationship with the lambda parameter $\lambda$ (i.e. the film thickness ratio).

$$
\lambda=\frac{h_{\min }}{\sqrt{R_{q A}^{2}+R_{q B}^{2}}}
$$

Since the lambda parameter $\lambda$ is designed to determine the effect of the contact conditions and the tribological 


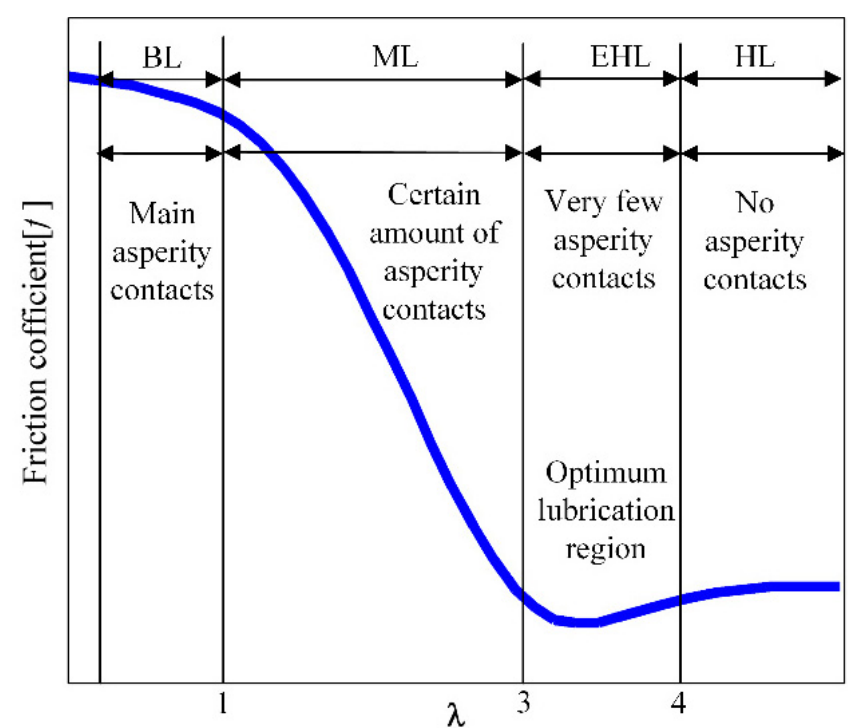

Fig. 4. Nature of friction coefficient and typical classification of different lubrication regimes depending on lambda parametre.

properties, it is typically used to determine the lubrication states that reasonably well demonstrate the amount of friction and the ability of contacts to separate the contacting surfaces. It can directly influence the friction coefficient and wear properties of a particular lubricating system.

The author draws the lubrication state diagram with comprehensive consideration of the existing models, which is schematically shown in Figure 4. Generally, the lubrication states, by using the lambda parameter, are divided as follows [34]: $\lambda \geq 3$ for hydrodynamic lubrication/elasto-hydrodynamic lubrication regime (HL/EHL), $1 \leq \lambda \leq 3$ for mixed lubrication regime (ML) and $\lambda \leq 1$ for boundary lubrication regime (BL).

A more precise classification also includes $\lambda \geq 4$ for fully $\mathrm{HL}$ and the bearing is in full film lubrication region with no asperity contacts. Mixed lubrication regime (ML) can also be divided up by $1 \leq \lambda \leq 1.5$ and $1.5 \leq \lambda \leq$ 3 , indicating the much asperity contacts and a certain amount of asperity contacts, respectively [34].

\subsection{Analysis of friction coefficient}

The typical Stribeck curve and the lubrication state configuration are shown in Figure 5, which is a schematic of the dependence of the overall friction on the contribution from "solid" and "viscous" forces. Among them, $X$ axis is the journal speed (Note: water film thickness is rather small and difficult to measure exact values, using speed instead of lambda), $Y$ axis is the friction coefficient $f$. F-solid (the red dashed line), is the solid-solid contact friction coefficient due to asperity contacts on the lubrication interface, namely "solid-solid effect", which increases with radial load according to Coulomb's law. Ffluid (the black dashed line), is the fluid friction coefficient

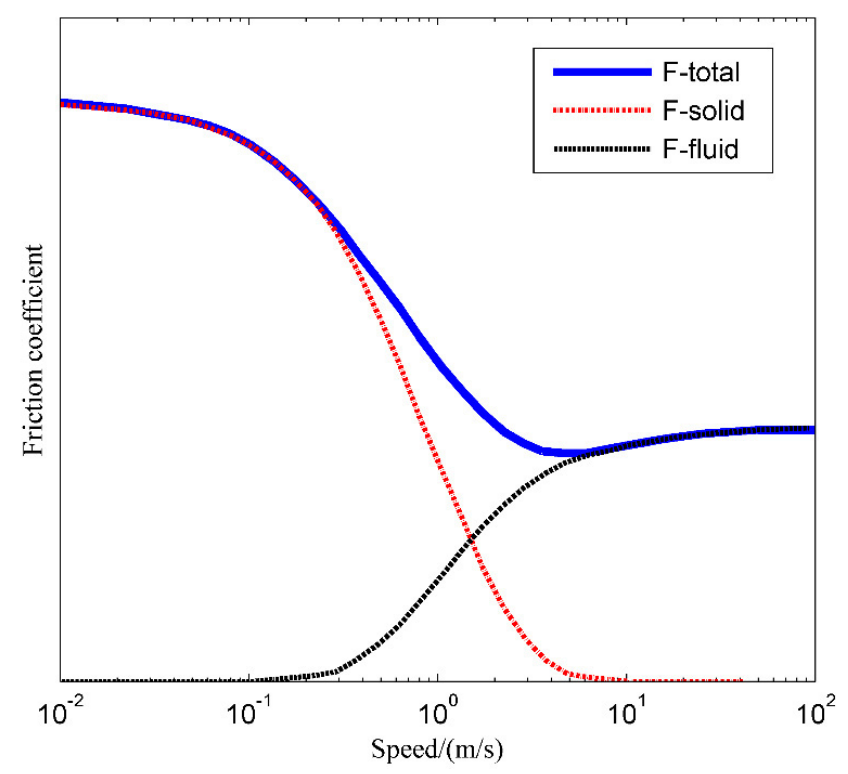

Fig. 5. Correlation between total friction coefficient and solid effect and viscous effect.

due to the viscous shear force between liquid molecules, namely "viscous effect". F-total (the blue thick line) is the total friction coefficient, the widely used Stribeck curve [15], which is the weighted sum between "solidsolid effect" and "viscous effect" as shown in Figure 5. Therefore, essence of friction coefficient is the linear combination of the solid-solid contact friction coefficient and the fluid viscous shear stress in water lubricated bearing.

We can see from Figure 5, under certain working conditions, the available formula of friction coefficient in water lubricated bearing is as follows:

$$
\begin{aligned}
& f_{\text {total }}=\alpha_{1} f_{\text {solid }}+\alpha_{2} f_{\text {fluid }} \\
& \alpha_{1}+\alpha_{2}=1, \alpha_{1}, \alpha_{2} \in[0,1]
\end{aligned}
$$

where, $\alpha_{1}$ is the weighted factor of "solid effect", $f_{\text {solid }}$ is the friction coefficient produced by "solid effect". The calculation method and procedure of $f_{\text {solid }}$ can be attained in references $[14,35] . \alpha_{2}$ is the weighted factor of "viscous effect", $f_{\text {fluid }}$ is the friction coefficient produced by "viscous effect". $f_{\text {fluid }}$ can be calculated by Equation (15).

Moreover, a change of the film thickness and the hydrodynamics will in turn affect the friction and the contact state again because of the modified contact conditions or even the lubrication state through increased solid-solid contacts. In fact, lubrication state is in a constant change in actual operation. The lubrication state transition changes correspondingly with the change of operating parameters. So, the film thickness ratio $\lambda$ is in a dynamic fluctuation state, causing the fluctuation of the friction coefficient. Note, under different working conditions, even the same values of friction coefficients, their compositions may be distinctly different from each other.

Under the low speed region, the "viscous effect" makes up a small fraction of the total friction coefficient while the "solid effect" is dominant, $\alpha_{1} \rightarrow 1, \alpha_{2} \rightarrow 0$, so 


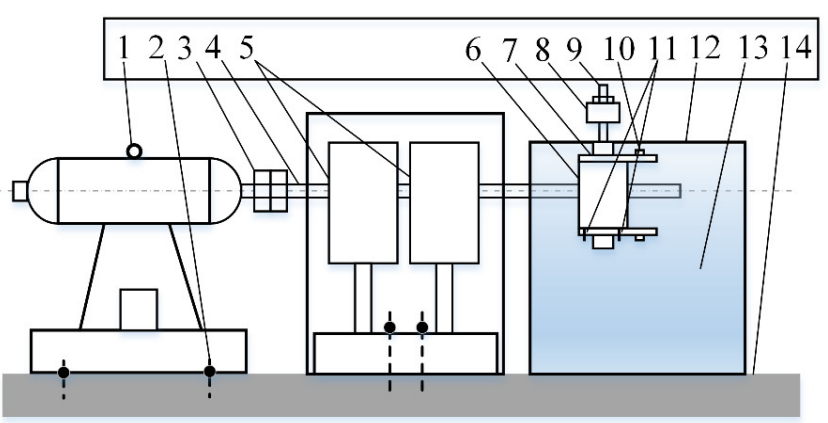

Fig. 6. Principle diagram of water lubricated bearing test rig 1 - servo motor; 2 - damper; 3 - coupling; 4 - shaft; 5 hydrostatic bearing; 6 - test bearing; 7 - sleeve; 8 - hydraulic cylinder; 9 - leading bar; 10 - force sensor; 11 - displacement transducer; 12 - water tank; 13 - water; 14 - base.

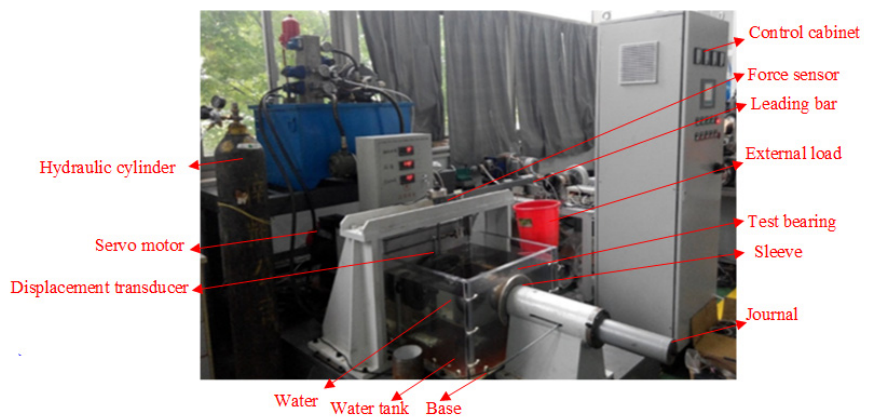

Fig. 7. Physical map of water lubricated bearing test rig.

the total friction coefficient curve is consistent with the F-solid curve in this region. Under high speed region, the fluid film can be fully formed, "viscous effect" is the leading factor while "solid effect" is insignificant, $\alpha_{1} \rightarrow 0, \alpha_{2} \rightarrow 1$, so the total friction coefficient curve is consistent with the F-fluid curve in this region. Therefore, there is a competition between the two physical effects, and friction coefficient will depend on the interplay between the "viscous" and "solid" effect contributions, as presented schematically in Figure 5.

\section{Experimental equipment}

\subsection{Introduction of test apparatus}

Figure 6 shows the schematic of the apparatus used for measuring the friction coefficient of water lubricated journal bearing, Figure 7 gives the physical map of the water lubricated bearing test rig, respectively.

The main shaft is supported by two hydrostatic bearings and driven by a Siemens servo motor with a maximum speed of $6000 \mathrm{rpm}$ and a maximum power of $22 \mathrm{~kW}$. The desired load is applied using a gas cylinder via a leading bar. The working pressure is usually about $0.3 \sim 0.8 \mathrm{MPa}$. The pipe resistance loss is rather small due to the low gas viscosity. It is also easy to focus on the supply and convenient for medium distance transportation. It is also safe to use, no explosion, no electric shock,

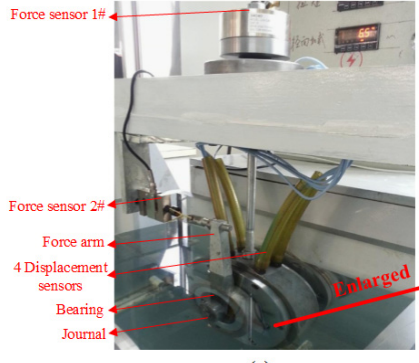

(a)

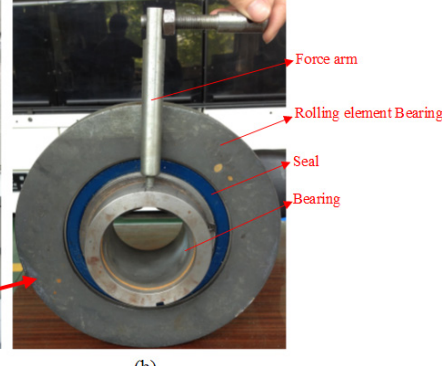

(b)
Fig. 8. Schematic diagram of the vertical loading part.

and the overload protection ability. The vertical loading lever scale is 1:5, i.e. when we apply $1 \mathrm{~N}$ load on the right side of lever, the applied load on the water lubricated bearing is magnified to maximum five times.

The experimental equipment is mainly consisted of the electric control part, the power-driven part, the intermediate part, the vertical loading part and the signal acquisition part. Figure 8 a shows the real object of measurement cell and vertical loading part in details. The lubrication grooves are not shown in Figure 8 for the sake of convenience. Components 8, 9, 10 and 11 form the main loading part, where force sensor $1 \#$ measures the vertical load.

Figure $8 \mathrm{~b}$ shows the enlarged figure of the measurement cell. Water lubricated bearing is embedded into the rolling element bearing. The functions of rolling element bearing are: (1) apply radial external load to the bearing; (2) help to measure the tangential force in order to calculate the friction torque and friction coefficient. A lever (i.e. the force arm) is fixed on the bearing surface. Therefore, the bearing would not rotate with the rotating journal. Force sensor $2 \#$ is mounted on the lever to measure the tangential force.

Figure 9a gives the simplified technical drawing of the whole bearing system, Figure $9 \mathrm{~b}$ shows the schematic of torque (force) equilibrium analysis for water lubricated bearing. When the journal is rotating, the rotating shaft drives the bearing through the friction force of water film, generates the driving torque $T_{f}$. The rolling element bearing and the force arm apply the torque $T_{R}, T_{F}$ in the opposite direction. Therefore, three torques exist for the bearing: torque $T_{f}$ due to the friction force of water film, torque $T_{R}$ due to the friction force of rolling element bearing, torque $T_{F}$ due to the force measured by force sensor $2 \#$. As can be seen from Figure 9b, we can get the torque equilibrium relationship. For water lubricated bearing, the equation of torque equilibrium is:

$$
T_{f}=T_{R}+T_{F}
$$

Generally, the diameter of the ball in rolling element bearing is small (less than $3 \mathrm{~mm}$, about $1 / 70$ of the force arm (the force arm of tangential force is $205 \mathrm{~mm}$ )), the friction force of rolling element bearing is small (friction coefficient of rolling element bearing is less than 0.001 , about one tenth of water lubricated bearing (friction coefficient is $10^{-2}$ order of magnitude for water lubrication 


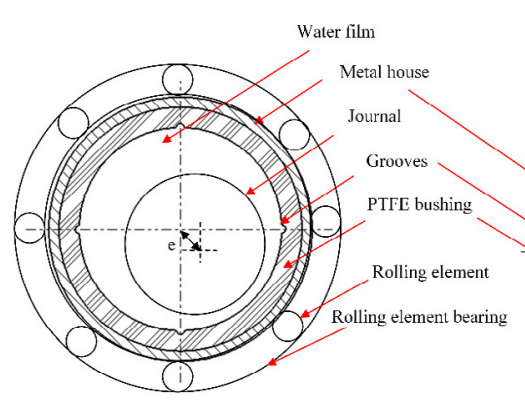

(a)

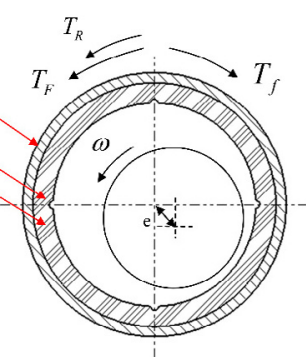

(b)
Fig. 9. Simplified technical drawing of the bearing.

Table 2. Basic parameters of water lubricated bearing.

\begin{tabular}{ccc}
\hline Description & Symbol & Value \\
\hline Width & $L$ & $20 \mathrm{~mm}$ \\
Diameter & $D$ & $62 \mathrm{~mm}$ \\
Clearance & $c$ & $0.07 \mathrm{~mm}$ \\
Bushing thickness & $T$ & $2 \mathrm{~mm}$ \\
Range of rotating speed & $N$ & $200-2000 \mathrm{rpm}$ \\
Range of load & $F$ & $8-62 \mathrm{~N}$ \\
\hline
\end{tabular}

Table 3. Water properties at $20{ }^{\circ} \mathrm{C}$.

\begin{tabular}{cc}
\hline Description & Value \\
\hline Saturation water vapor pressure & $2340 \mathrm{~Pa}$ \\
Saturation density of water & $998.2 \mathrm{~kg} . \mathrm{m}^{-3}$ \\
Saturation density of water vapor & $0.5542 \mathrm{~kg} . \mathrm{m}^{-3}$ \\
Dynamic viscosity of water & $1 \times 10^{-3} \mathrm{~Pa} \cdot \mathrm{s}$ \\
Dynamic viscosity of water vapor & $1.34 \times 10^{-5} \mathrm{~Pa} \cdot \mathrm{s}$ \\
\hline
\end{tabular}

bearing)). Therefore, torque $T_{R}$ due to the rolling element bearing is very small, and can be omitted in the actual calculation. Then we can get $T_{f} \approx T_{F}$, and torque $T_{F}$ is due to the force measured by force sensor $2 \#$. It equals to the product of the force arm $R$ and tangential force $T$. Force sensor $2 \#$ measures the tangential force $T$, then measures the arm $(R)$ of force applied on the bearing through the lever. The torque can be calculated by multiplied the tangential force $T$ and the force arm $R$.

In the actual tests, the friction coefficient is translated into tension or compression of a linkage bar and sensed by a loading cell, and the test signal is transferred to the computer system for recording and processing. The friction coefficient, the speed and the load are processed by software and displayed on the computer screen. The time interval of data reading is adjustable and is independent of the duration of the test.

\subsection{Test bearing}

Table 2 gives the basic parameters and operating conditions of the test bearing, Table 3 shows the basic physical properties of water in $20{ }^{\circ} \mathrm{C}$ environment.

Figure 10a shows the three dimensional model of the test bearing, Figure 10b shows the physical map of the test bearing. The material of the sleeve is the same as

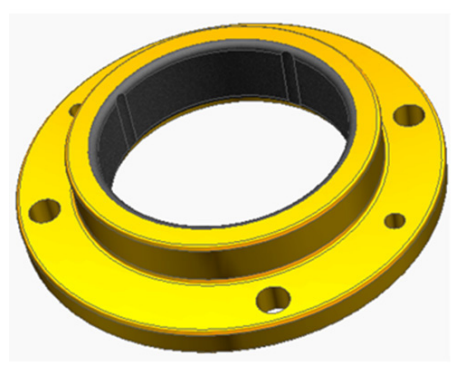

(a) (b)

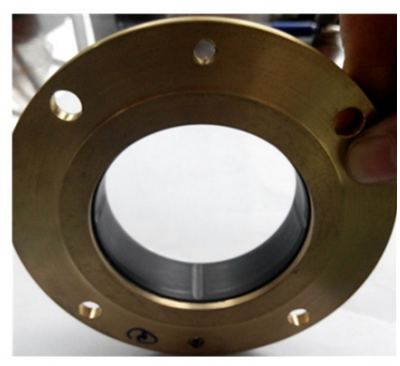

Fig. 10. Three dimensional model and physical map of test bearing.

shaft, and the bushing is made of composite material PTFE, which is highly resistant to impact, wear and corrosion and has adequate bearing capacity.

As a case, the test bearing is composed by the bushing and the sleeve, which are resistant to wear and corrosion. The shaft is made of hardened $42 \mathrm{CrMo}$ steel with 0.3 Poisson's ratio, 206 GPa elastic modulus. The surface finish (Ra) is about $0.4 \mu \mathrm{m}$ consistently for the shaft. The test bearing has an inner diameter $62 \mathrm{~mm}$, and the bushing thickness $2 \mathrm{~mm}$. It has 4 guiding grooves, its crosssection shape is round. The inner surface finish $(\mathrm{Ra})$ is $0.4 \mu \mathrm{m}$ consistently for the bushing. The bearing width is $20 \mathrm{~mm}$, therefore the length over diameter ratio is only about 0.33 , and the radial clearance is $0.07 \mathrm{~mm}$, about two thousandth of the shaft radius. The specific values of the bearing and water properties may refer to Tables 2 and 3 , respectively.

Figure 11 gives the surface structure and fracture of the test specimen of the composite material PTFE. Figure 11a shows the uniform distribution of dense white spots on the tissue surface observed by high power microscope, these white spots are mainly consist of high wearresistant engineering PTFE material particles. Figure 11b shows the fracture morphology of the test specimen, highstrength fiber uniformly distributed in the PTFE material tissue, and formed disordered arranged crisscross patterns, which can significantly improve the shear-resistant, compressive and wear-resistant properties.

\subsection{Experimental conditions and method}

The experimental parameters and operating conditions are shown in Tables 2 and 4 . Group 1 investigates the effect of vertical load on the friction coefficient under different rotating speeds, and obtains the results for the friction coefficient as the function of vertical load ranging from 8-62 N; Group 2 researches the effect of rotating speed on the friction coefficient under fixed vertical load, and obtains the curve for the friction coefficient as the function of rotating speed ranging from 200-3000 rpm.

Before any measurement is taken, the test system should be balanced so that the friction coefficient is zero when the shaft is at a static position. At each speed, the history of the friction coefficient is monitored. The rms 
Table 4. Experimental conditions of water lubricated bearing.

\begin{tabular}{cccc}
\hline Group & $\begin{array}{c}\text { Operating } \\
\text { condition }\end{array}$ & Value & Increment \\
\hline \multirow{2}{*}{ Group 1 } & $\begin{array}{c}\text { Range of load } \\
\text { Range of speed }\end{array}$ & $\begin{array}{c}8-62 \mathrm{~N} \\
400-2000 \mathrm{rpm}\end{array}$ & $\begin{array}{c}\approx 10 \mathrm{~N} \\
200 \mathrm{rpm}\end{array}$ \\
\hline \multirow{2}{*}{ Group 2 } & $\begin{array}{c}\text { Fixed load } \\
\text { Range of speed }\end{array}$ & $\begin{array}{c}32 \mathrm{~N} \\
200-3000 \mathrm{rpm}\end{array}$ & $\begin{array}{c}- \\
200 \mathrm{rpm}\end{array}$ \\
\hline
\end{tabular}

value of the history is taken as the friction coefficient for the specified speed when the friction coefficient oscillates periodically around a relatively constant value. By plenty of observations, a period of $3 \mathrm{~min}$ is regarded as a reasonable testing period for each vertical load point or each rotating speed.

In the test, the parameters need to measure includes: the bearing radial force measured by the force sensor $1 \#$, the rotating speed $N$, the tangential force $T$ measured by the force sensor $2 \#$, the arm of force applied on the bearing through the lever $R$, the bearing inner diameter $D$, the width $L$ and the gravity $G$ of the bearing. The test apparatus is shown in Figure 8.

Therefore, the radial load can be calculated as:

$$
F r=F-G
$$

Correspondingly, the friction coefficient can be calculated as:

$$
f=\frac{2 T \times R}{F r \times D}
$$

where, the specific parameters of the test bearing can be checked in Table 2, $R=205 \mathrm{~mm}, D=62 \mathrm{~mm}, G=15 \mathrm{~kg}$, insert them into Equations (20) and (21), then we can get:

$$
f=\frac{205 T}{31(F-G)}=\frac{205 T}{31 F r}
$$

\section{Results and discussion}

Additional parameters used in the experiments are provided in Table 4.

\subsection{Analysis of friction coefficient fluctuation}

Figure 12 shows the friction coefficient distributions as the function of radial load at different rotating speeds $200 \mathrm{rpm}, 400 \mathrm{rpm}$ and $1600 \mathrm{rpm}$. These three rotating speeds represent three typical operating conditions: low, medium and high speed region, respectively. And, $X$ axis represents the radial load applied on water lubricated bearing, $Y$ axis represents the measured friction coefficients. The friction coefficient curves contain the error bars, i.e. the I-shaped errors, which reflect the standard deviations (i.e. the average deviation).

From Figure 12 we can see, for different rotating speeds, friction coefficient curves as the function of load exhibit consistency on the overall trend, but also exist discrepancies in some details. For example, the minimum

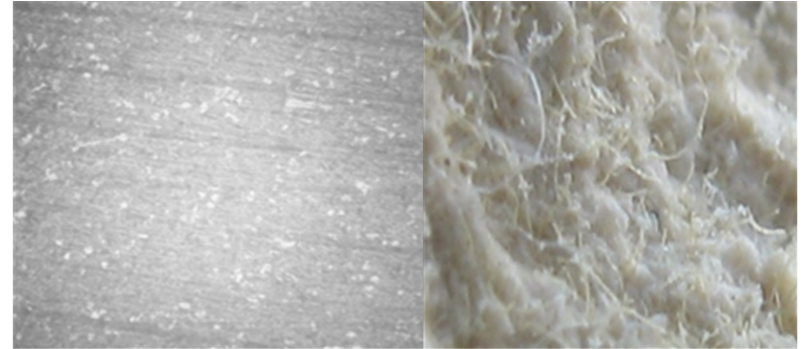

(a)

(b)

Fig. 11. Surface structure and fracture of the composite material PTFE.

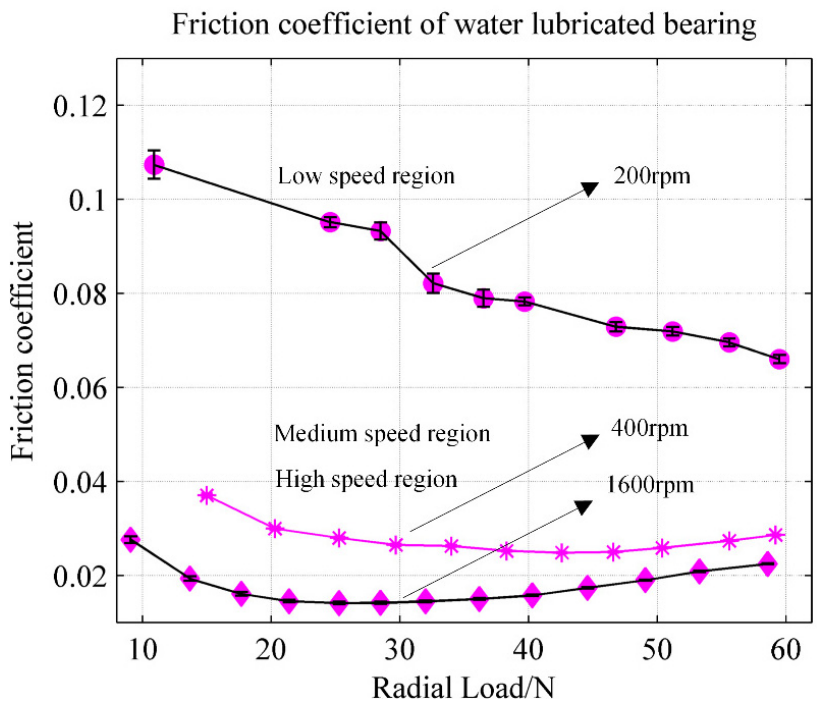

Fig. 12. Friction coefficient of water lubricated bearing (rotating speed $200 \mathrm{rpm}, 400 \mathrm{rpm}, 1600 \mathrm{rpm}$ ).

friction coefficient takes place at different radial loads. When the radial load is very small, friction coefficient is rather large. With increase of radial load, friction coefficient decreases mildly. But when the load increases to a certain value, friction coefficient tends to be stable. Continue to increase the load, friction coefficient rises slowly, and the higher the rotating speed, the more obvious the increasing trend.

In fact, main reason for the fluctuation of friction coefficient comes from two aspects: (1) competitions between the two physical effects: "solid effect" and "viscous effect", and friction coefficient depends on the interplay between the "solid" and "viscous" friction contributions, as presented schematically in Figure 5. (2) Changes of lubrication states of the composite material bushing caused by the elastic deformation under different speed and load conditions. Although the overall trend of friction coefficient curve is the same, the concrete mechanism differs for different rotating speeds. In order to analyze the mechanism clearly, we divide friction coefficient curve into three regions: low, medium and high speed region.

In the low speed region (about 0-400 rpm), the bearing loading capacity is relatively small, and elastic deformation of the bushing plays a major role. The water film thickness is rather thin in the lubrication region, bearing 
is in the boundary lubrication regime (BL). Accordingly, asperity contacts between the rough surfaces dominate and determine the type of friction and wear, and the "solid effect" makes the major contributions to the friction coefficient of the bearing. With the increase of radial load, elastic deformation is larger and larger, so necking phenomenon of the water film is more and more obvious. Then, the shaft is slowly floated by the increasing thick film, the bearing loading area as well as the lubrication region area increases, and thus decreases the friction coefficient. When the load increases to a certain value, the elastic deformation reaches to its maximum value, the bearing loading area as well as the lubrication region area no longer changes. Continue to increase radial load, friction coefficient tends to be stable gradually.

In the high speed region (about 1200-2000 rpm), water film can be sufficiently formed. The bearing is fully lubricated and in the hydrodynamic lubrication state. Therefore, when the load is small, the "viscous effect" dominates and the viscous shear force between the liquid molecules constitutes the main part of friction coefficient. As with the increase of radial load, the film thickness decreases gradually, and the lubrication state transforms from hydrodynamic lubrication regime (HL) to elasto-hydrodynamic lubrication regime (EHL), mixed lubrication regime (ML) and even the boundary lubrication regime $(\mathrm{BL})$. In this process, the proportion of "viscous effect" declined slowly, friction coefficient also decreases gradually and reaches the minimum value finally. Afterwards, continue to increase radial load, the lubrication state transforms to the boundary lubrication regime (BL) or even the dry friction regime, the proportion of "viscous effect" continues to reduce until it can be almost negligible while the proportion of the "solid effect" rises correspondingly until it becomes dominant. Therefore, friction coefficient begins to rise in the process and then increases with the increasing radial load.

For medium speed region (400-1200 rpm), friction coefficient is determined by the weighted factors of "solid effect" and "viscous effect" $\alpha_{1}, \alpha_{2}$, as well as the elastic deformation of the PTFE bushing. Therefore, characteristics of the curve in the medium speed region is somewhere between the characteristics of low speed region and the characteristics of high speed region, almost the linear combination of the typical low speed curve and typical high speed curve.

\subsection{Analysis of the system limitations}

From Figure 12 we can also see, the error bars (i.e. the I-shaped error) become smaller and smaller with the rise of load under fixed speed. That is to say, the fluctuation of the measured friction coefficients is smaller and smaller. When radial load increases to a certain value, the I-shaped error is almost close to zero, the friction coefficient curve has almost no fluctuation, and is stable at a fixed value.

Generally speaking, fluctuation of the friction coefficient is mainly caused by the oscillation of the weighted factors of "solid effect" and "viscous effect" due to the

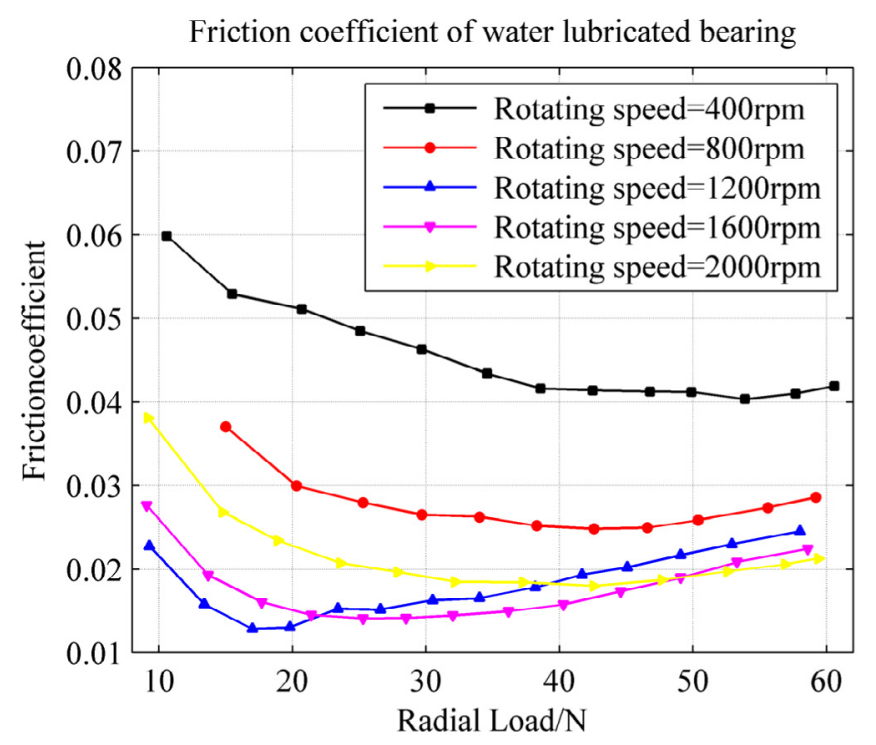

Fig. 13. Friction coefficient under different rotating speeds.

transition of the lubrication state. The smaller the fluctuation of friction coefficient, the more stable the bearing operating conditions. Fluctuation is relatively small under high speed condition for the "viscous effect" dominates and the "solid effect" is almost ignorable; Fluctuation is relatively large under low speed condition for sufficient lubrication of the bearing cannot be ensured and "solid effect" dominates the lubrication state, "viscous effect" is almost ignorable. There exists large number of asperity contacts between rough surfaces in low speed lubrication region. It is the asperity contact that cause the fluctuation of the friction coefficients.

Of course, system limitations exist and influence the exact values of friction coefficient. Precision error of force sensors $1 \#$ and $2 \#$ also affects the fluctuations of friction coefficient. Disadvantages of pneumatic system (the low pressure transmission speed, the long reaction time, poor speed stability due to the compressibility of the air) bring rather big impacts on the control accuracy of the system. For the external load, it is prone to be unstable. Numerical fluctuations for the load values exist in the specific test process. Therefore, the next step, we will use the mechanical loading device instead of the hydraulic cylinder, because the mechanical loading device has better stability.

\subsection{Effect of radial load}

Figure 13 shows the friction coefficient distribution as the function of radial load at different rotating speeds, and $X$ axis represents the radial load applied on the water lubricated bearing, $Y$ axis represents the measured friction coefficients.

From Figure 13 we can see, under fixed rotating speed, friction coefficient curves show first decreased and then increased trend with the increase of the radial load. There exists an appropriate working condition which the friction 
Friction coefficient of water lubricated bearing

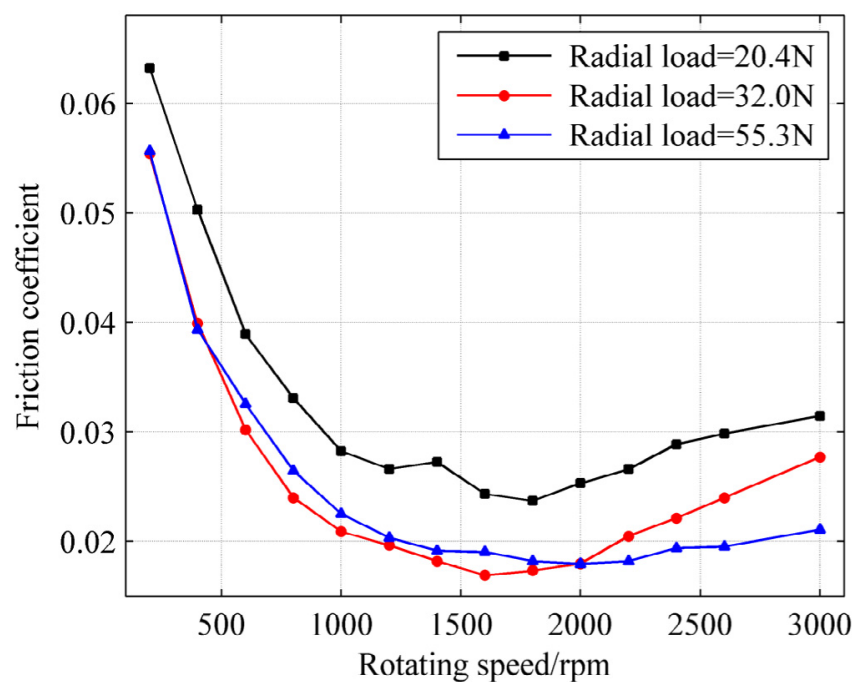

Fig. 14. Friction coefficient under different radial loads.

coefficient is minimum. Generally speaking, this condition is the ideal state for the bearing operation.

For constant low load, bearing surface deformation is rather small, the bearing is in hydrodynamic lubrication state. With the increasing load, the hydrodynamic film becomes partially breakdown and thus results in the increase of surface roughness peak contact, namely the asperity contact. Continue to increase the load, the bearing bushing surface elastic deformation becomes distinct, surface roughness peak contact area becomes larger and larger. Correspondingly, the load per unit area becomes smaller and smaller. Therefore, friction coefficient increases slowly and will become constant when the radial load reaches critical value. The interaction of the two factors results in the existence of minimum value of friction coefficient under certain speed.

\subsection{Effect of rotating speed}

Figure 14 shows the friction coefficient distribution as the function of rotating speed at different radial loads, and $X$ axis represents the rotating speed of the shaft, $Y$ axis represents the measured friction coefficients.

From Figure 14 we can see, the measured friction coefficient-rotating speed curves agree well with the classical Stribeck curve. For different radial loads, friction coefficient curves exhibit consistency of the overall trend, but also exist some discrepancies in details. Under fixed load, obviously, with the increase of rotating speed, the friction coefficient first decreases sharply in the low speed region (0-1200 rpm), decreases slowly in the medium and high speed region (1200-2200 rpm), and then increases mildly in the very high speed region (2200-3000 rpm).

Radial load has influence on the friction coefficient. This is because, under low speed region, the bearing is in boundary lubrication state and "solid effect" predominates the motion, while the bearing is in full partial

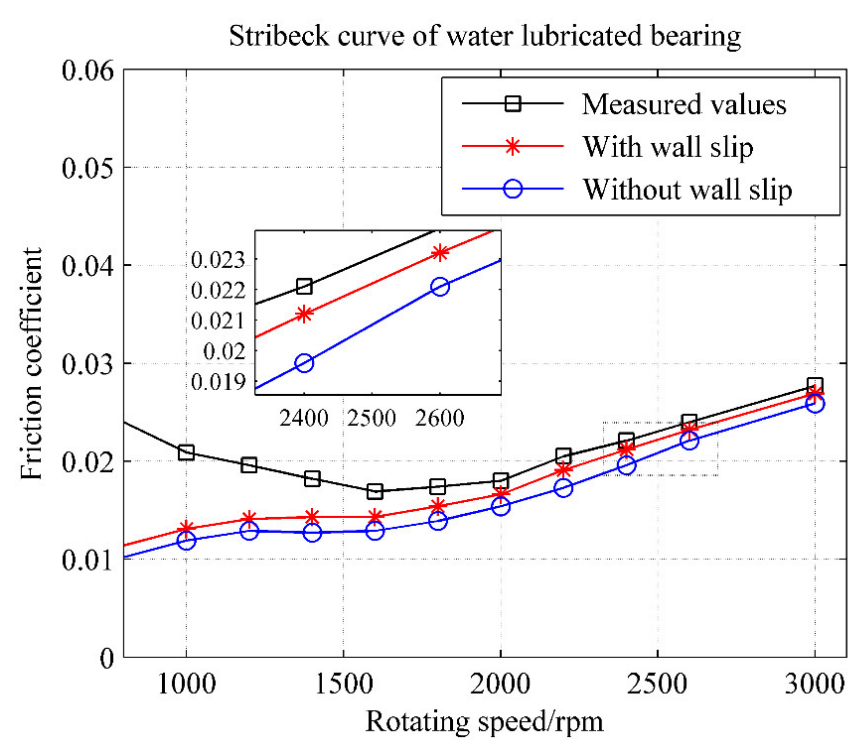

Fig. 15. Contrast curve between theoretical and experimental friction coefficient.

hydrodynamic lubrication state and "viscous effect" predominates the motion under high speed region. The friction coefficient curve increases more moderately when the radial load is $55.3 \mathrm{~N}$. The interaction of the two factors results in the existence of minimum value of friction coefficient under certain load.

\subsection{Comparison of experimental and theoretical results}

Figure 15 shows the measured and theoretical calculated friction coefficient distribution as the function of rotating speed, $X$ axis represents the rotating speed of the shaft, $Y$ axis represents the friction coefficients. "Measured values" curve represents the results measured in the test; "With wall slip" represents the results considering the wall slip boundary condition; "Without wall slip" represents the results without considering the wall slip boundary condition. The radial load in the test is $32.0 \mathrm{~N}$.

From Figure 15 we can see, with the increase of rotating speed, the measured friction coefficients gradually decrease, reach its minimum value at $1600 \mathrm{rpm}$, and then slowly increase. Just as the theory predicted in Section 2.3, the theoretical values gradually increase and become more and more close to the measured values with increase of rotating speed. The difference between the measured and theoretical values is smaller and smaller, and eventually reaches zero when rotating speed increases to a certain value. Results considering wall slip are much closer to the measured values. The higher is the rotating speed, the smaller is the deviation between them. In fact, when the rotating speed exceeds $2200 \mathrm{rpm}$, errors between the measured and "With wall slip" results are rather small, almost ignorable. This consistence indicates that there is a real possibility that wall slip effect exists in the lubrication interface of water lubricated PTFE bearing. And, the higher is the rotating speed, the larger 
is the possibility of existence of wall slip. This conclusion is consistent with the previous achievements in references $[19,23,25,29,36]$. The modified model is more accurate in the prediction of friction coefficient for water lubricated PTFE bearing.

\section{Conclusions}

In this paper, on the basement of experimental results of water lubricated composite material bearing, we measure the friction coefficient as the function of load and rotating speed, deviate the modified Reynolds equation considering wall slip effects in Cartesian coordinate system. We develop essential model of friction coefficient, discuss the composition of friction coefficient and the changing correlation between components of friction coefficient, and finally draw the following conclusions:

(1) The essence of friction coefficient in water lubricated bearing is the weighted sum of solid contact friction coefficient and viscous shear stress between liquid molecules. Lubrication state is in a constant change in actual operation. The transition of lubrication state changes correspondingly with the operating parameters. Film thickness ratio $\lambda$ is in a dynamic fluctuation state, causing the fluctuation of friction coefficient.

(2) There is always an appropriate working condition which the friction coefficient is minimum, and this condition is the ideal state for the bearing operation.

(3) Under fixed rotating speed, with increase of radial load, the friction coefficient first decreases and then rises, strikes its minimum value under a certain load. Similarly, under fixed load, with increase of rotating speed, the friction coefficient first decreases and then rises, reaches its minimum value under a certain rotating speed.

(4) The difference between the friction coefficient measured by the test and the friction coefficient calculated by the theory is just the friction coefficient generated by the solid-solid interface contact effect. As the rotating speed increases, the friction coefficient produced by the "solid effect" will become smaller and smaller, and finally approaches zero under a certain rotating speed.

(5) Theoretical results considering wall slip are in good agreement with the measured values. The higher is the rotating speed, the smaller is the deviation between them. This phenomenon verifies the existence of wall slip effects in the interface of water lubricated bearing and also verifies the correctness of the theoretical model.

Acknowledgements. This research receives no specific grant from any funding agency in the public, commercial, or notfor-profit sectors.

\section{References}

[1] D. Cabrera, et al., Film pressure distribution in waterlubricated rubber journal bearings, Proc. Inst. Mech. Eng. 219 (2005) 125-132
[2] P. Andersson, P. Lintula, Load-carrying capability of water-lubricated ceramic journal bearings, Tribol. Int. 27 (1994) 315-21

[3] M.M. Maru, et al., The Stribeck curve as a suitable characterization method of the lubricity of biodiesel and diesel blends, Energy 69 (2014) 673-681

[4] I. Faraon, D. Schipper, Stribeck curve for starved line contacts, J. Tribol. Trans. ASME 129 (2007) 181-187

[5] X.B. Lu, E. Gelinck, M. Khonsari, The Stribeck curve: experimental results and theoretical prediction, J. Tribol. Trans. ASME 128 (2006) 789-794

[6] E. Gelinck, D. Schipper, Calculation of Stribeck curves for line contacts, Tribol. Int. 33 (2000) 175-181

[7] M. Kalin, I. Velkavrh, Non-conventional inverse-Stribeckcurve behaviour and other characteristics of DLC coatings in all lubrication regimes, Wear 297 (2013) 911-918

[8] J. Wang, F. Yan, Q. Xue, Tribological behavior of PTFE sliding against steel in sea water, Wear 267 (2009) 16341641

[9] W. Huang, et al., The tribological performance of $\mathrm{Ti}(\mathrm{C}, \mathrm{N})$-based cermet sliding against Si3N4 in water, Wear 270 (2011) 682-687

[10] X. Lei, et al., Tribological behavior between micro- and nano-crystalline diamond films under dry sliding and water lubrication, Tribol. Int. 69 (2014) 118-127

[11] M. Masuko, et al., Friction characteristics of inorganic or organic thin coatings on solid surfaces under water lubrication, Tribol. Int. 39 (2006) 1601-1608

[12] C. Min, et al., Study of tribological properties of polyimide/graphene oxide nanocomposite films under seawater-lubricated condition, Tribol. Int. 80 (2014) 131140

[13] A. Abdelbary, et al., The effect of surface defects on the wear of Nylon 66 under dry and water lubricated sliding, Tribol. Int. 59 (2013) 163-169

[14] A. de Kraker, R.A.J. van Ostayen, D.J. Rixen, Calculation of Stribeck curves for (water) lubricated journal bearings, Tribol. Int. 40 (2007) 459-469

[15] M. Kalin, I. Velkavrh, J. Vižintin, The Stribeck curve and lubrication design for non-fully wetted surfaces, Wear 267 (2009) 1232-1240

[16] D.C. Tretheway, C.D. Meinhart. Apparent fluid slip at hydrophobic microchannel walls, Phys. Fluid 14 (2002) L9-L12

[17] C.-H. Choi, K.J.A. Westin, K.S. Breuer, Apparent slip flows in hydrophilic and hydrophobic microchannels, Phys. Fluid 15 (2003) 2897-2902

[18] C. Neto, et al., Boundary slip in Newtonian liquids: a review of experimental studies, Rep. Prog. Phys. 68 (2005) 2859

[19] C. Neto, V Craig, D. Williams, Evidence of sheardependent boundary slip in Newtonian liquids, Eur. Phys. J. E 12 (2003) 71-74

[20] O.I. Vinogradova, Slippage of water over hydrophobic surfaces, Int. J. Miner. Process. 56 (1999) 31-60

[21] S. Granick, Y. Zhu, H. Lee, Slippery questions about complex fluids flowing past solids, Nat. Mater. 2 (2003) 221227

[22] H.A. Spikes, The half-wetted bearing. Part 1: extended Reynolds equation, Proc. Inst. Mech. Eng. 217 (2003) 114

[23] F. Aurelian, M. Patrick, H. Mohamed, Wall slip effects in (elasto) hydrodynamic journal bearings, Tribol. Int. 44 (2011) 868-877 
[24] H. Zhang, et al., Boundary slip surface design for high speed water lubricated journal bearings, Tribol. Int. 79 (2014) 32-41

[25] F. Guo, et al., Occurrence of wall slip in elastohydrodynamic lubrication contacts, Tribol. Lett. 34 (2009) 103111

[26] C.W. Wu, Performance of hydrodynamic lubrication journal bearing with a slippage surface, Ind. Lubr. Tribol. 60 (2008) 293-298

[27] S. Hatzikiriakos, J. Dealy, Wall slip of molten high density polyethylene. I. Sliding plate rheometer studies, J. Rheol. 35 (1991) 497-523

[28] S.G. Hatzikiriakos, J.M. Dealy, Wall slip of molten high density polyethylenes. II. Capillary rheometer studies, J. Rheol. 36 (1992) 703-741

[29] M. Kaneta, H. Nishikawa, K. Kameishi, Observation of wall slip in elastohydrodynamic lubrication, J. Tribol. Trans. ASME 112 (1990) 447-452
[30] S. Richardson, On the no-slip boundary condition, J. Fluid. Mech. 59 (1973) 707-719

[31] T. Chung, Computational fluid dynamics, Cambridge University Press, New York, 2010

[32] A.Z. Szeri, Fluid film lubrication: theory and design, Cambridge University Press, New York, 2005

[33] A.Z. Szeri, Fluid film lubrication, Cambridge University Press, New York, 2011, Vol. 2

[34] G. Stachowiak, A.W. Batchelor, Engineering Tribology, Butterworth-Heinemann, Oxford, 2013

[35] A. Fatu, D. Bonneau, R. Fatu, Computing hydrodynamic pressure in mixed lubrication by modified Reynolds equation, Proc. Inst. Mech. Eng. 226 (2012) 1074-1094

[36] G.J. Ma, C.W. Wu, P. Zhou, Influence of wall slip on the hydrodynamic behavior of a two-dimensional slider bearing, Acta Mech. Sin. 23 (2007) 655-661 